\title{
The Flying Saucer: Tomography of the thermal and density gas structure of an edge-on protoplanetary disk
}

\author{
A. Dutrey ${ }^{1}$, S. Guilloteau ${ }^{1}$, V. Piétu ${ }^{2}$, E. Chapillon ${ }^{1,2}$, V. Wakelam ${ }^{1}$, E. Di Folco ${ }^{1}$, T. Stoecklin ${ }^{3}$, O. Denis-Alpizar ${ }^{4}$,

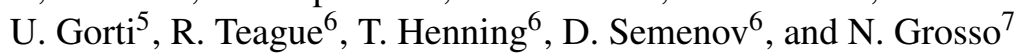 \\ ${ }^{1}$ Laboratoire d'astrophysique de Bordeaux, Univ. Bordeaux, CNRS, B18N, Allée Geoffroy Saint-Hilaire, 33615 Pessac, France \\ e-mail: anne.dutrey@u-bordeaux.fr \\ 2 IRAM, 300 rue de la piscine, 38406 Saint Martin d'Hères, France \\ 3 Institut des Sciences Moléculaires, UMR5255-CNRS, 351 Cours de la libération, 33405 Talence, France \\ 4 Universidad Autónoma de Chile, Instituto de Ciencias Quimicas Aplicadas, Theoretical and Quantum Chemistry Center, \\ 2801 El Llano Subercaseaux, San Miguel, Santiago, Chile \\ 5 SETI Institute/NASA Ames Research Center, Mail Stop 245-3, Moffett Field, CA 94035-1000, USA \\ ${ }^{6}$ Max-Planck-Institute für Astronomie, Königstuhl 17, 69117 Heidelberg, Germany \\ 7 Observatoire Astronomique de Strasbourg, Université de Strasbourg, CNRS, UMR 7550, 11 rue de l'Université, 67000 Strasbourg, \\ France
}

Received 17 February 2017 / Accepted 5 June 2017

\begin{abstract}
Context. Determining the gas density and temperature structures of protoplanetary disks is a fundamental task in order to constrain planet formation theories. This is a challenging procedure and most determinations are based on model-dependent assumptions. Aims. We attempt a direct determination of the radial and vertical temperature structure of the Flying Saucer disk, thanks to its favorable inclination of 90 degrees.

Methods. We present a method based on the tomographic study of an edge-on disk. Using ALMA, we observe at $0.5^{\prime \prime}$ resolution the Flying Saucer in CO $J=2-1$ and CS $J=5-4$. This edge-on disk appears in silhouette against the CO $J=2-1$ emission from background molecular clouds in $\rho$ Oph. The combination of velocity gradients due to the Keplerian rotation of the disk and intensity variations in the $\mathrm{CO}$ background as a function of velocity provide a direct measure of the gas temperature as a function of radius and height above the disk mid-plane.

Results. The overall thermal structure is consistent with model predictions, with a cold $(<12-15 \mathrm{~K})$ CO-depleted mid-plane and a warmer disk atmosphere. However, we find evidence for CO gas along the mid-plane beyond a radius of about 200 au, coincident with a change of grain properties. Such behavior is expected in the case of efficient rise of UV penetration re-heating the disk and thus allowing CO thermal desorption or favoring direct CO photo-desorption. CO is also detected at up to 3-4 scale heights, while CS is confined to around 1 scale height above the mid-plane. The limits of the method due to finite spatial and spectral resolutions are also discussed.

Conclusions. This method appears to be a very promising way to determine the gas structure of planet-forming disks, provided that the molecular data have an angular resolution which is high enough, on the order of $0.3-0.1^{\prime \prime}$ at the distance of the nearest star-forming regions.
\end{abstract}

Key words. circumstellar matter - protoplanetary disks - radio lines: stars

\section{Introduction}

Protoplanetary disks orbiting young pre-main sequence stars are the sites of planetary system formation. In these disks, gas represents about $99 \%$ of the mass and is mostly in the form of $\mathrm{H}_{2}$. A minimum mass of $0.01 M_{\odot}$ was determined by Weidenschilling (1977) for the protosolar nebula based on the current solar system; since then models of planetary system formation have evolved drastically. Observational determinations of vertical and radial mass distribution of protoplanetary disks provide key constraints for planet formation models. Furthermore, studying the gas and dust distributions in protoplanetary disks found around low-mass T Tauri stars, recognized as young analogs to the solar system, has become a major challenge to understanding how planetary systems form and evolve.
With the advent of ALMA, many new results such as the observation of narrow dust rings in the HL Tau dust disk (ALMA Partnership et al. 2015) are changing our views on these objects. A series of studies of the disk associated with TW Hydrae - the closest T Tauri star - has significantly improved our understanding of disk physics and chemistry. This disk is seen almost face-on, maximizing its surface, and the dust and gas distributions have been intensively observed and modeled (Qi et al. 2004, 2013; Andrews et al. 2012, 2016; Rosenfeld et al. 2012; van Boekel et al. 2017; Teague et al. 2017). The $J=1-0$ transition of HD, the hydrogen deuteride, has been also detected by Bergin et al. (2013) who determined the gas mass of the disk to be $>0.056 M_{\odot}$, a value ranging at the upper end of previous estimates based on indirect mass tracers $\left(5 \times 10^{-4}\right.$ $0.06 M_{\odot}$; Thi et al. 2010; Gorti et al. 2011). More recently 
Teague et al. (2016) used simple molecules such as CO, CN, or $\mathrm{CS}$ to determine the turbulence inside the disk and found that the turbulent line broadening is less than $0.05 \mathrm{~km} \mathrm{~s}^{-1}$. Schwarz et al. (2016) reanalyzed the HD observations and confirmed the high mass of the disk. However, both studies are limited by the knowledge of the disk's vertical structure, in particular the thermal profile of the gas, and assumptions have to be made on the vertical location of molecules since this cannot be directly recovered in a face-on disk.

Contrary to a face-on object, the disk around HD 163296, a Herbig Ae star of $2 M_{\odot}$, is inclined by about $45^{\circ}$ along the line of sight. Such an inclination is enough to partially reveal the vertical location of the molecular layer, confirming that a significant fraction of the mid-plane is devoid of $\mathrm{CO}$ emission (de Gregorio-Monsalvo et al. 2013; Rosenfeld et al. 2013). In the case of IM Lupi, a $1 M_{\odot}$ star surrounded by a disk inclined by about $45^{\circ}$, a multi-line $\mathrm{CO}$ analysis coupled to a study of the dust disk (images and SED) allowed Cleeves et al. (2016) to provide a coherent picture of the gas and dust disk. However, owing to the combination of Keplerian shear and inclination, a complete determination of the vertical structure is challenging because at a given velocity there can be several locations (radii) inside the disk (see Beckwith \& Sargent 1993). In other words, there is no perfect correspondence between a radius and a velocity and this generates degeneracies, which are particularly important when the spatial resolution is limited. A purely edge-on disk can allow the retrieval of the full vertical structure of the molecules from which the density and temperature vertical gradients can be derived, provided the angular resolution is high enough and the molecular transitions are adequately selected.

To test the ability of deriving the disk vertical structure from an edge-on disk, we submitted the ALMA project 2013.1.00387.S dedicated to the study of the Flying Saucer. The Flying Saucer (2MASS J16281370-2431391) is an isolated, edge-on disk in the outskirts of the $\rho$ Oph dark cloud L 1688 (Grosso et al. 2003) with evidence for 5-10 $\mu \mathrm{m}$-sized dust grains in the upper layers (Pontoppidan et al. 2007). Grosso et al. (2003) resolved the light scattered by micron-sized dust grains in the near-infrared with the ESO New Technology Telescope (NTT) and the Very Large Telescope (VLT) and estimated from the nebula extension dust a disk radius of $2.15^{\prime \prime}$, which is about $260 \mathrm{au}$ for the adopted distance of $120 \mathrm{pc}$ (Loinard et al. 2008). The detection of the $\mathrm{CN} \mathrm{N}=2-1$ line (Reboussin et al. 2015) indicated the presence of a large gas disk. The $\rho$ Oph region is crowded with molecular clouds that are strongly emitting in $\mathrm{CO}$ lines. The low extinction derived by Grosso et al. (2003) toward the Flying Saucer suggests that it lies in front of these clouds, and this is confirmed by the CO study of Guilloteau et al. (2016).

We observed CO $J=2-1$, CS $J=5-4$, and CN $N=2-1$. This is a set of standard lines which has been extensively used to retrieve disk structures (Dartois et al. 2003; Piétu et al. 2007; Chapillon et al. 2012; Rosenfeld et al. 2013).

The dust and $\mathrm{CO}$ emissions detected in this ALMA project were partly discussed in Guilloteau et al. (2016) where we analyzed the absorption of the $\mathrm{CO}$ background cloud by the dust disk, deriving a dust temperature of about $7 \mathrm{~K}$ in the dust disk mid-plane at $100 \mathrm{au}$. This second paper deals with the retrieval of the gas temperature and density structures based on the analysis of the low angular resolution CO and CS lines. After showing the results and the analysis of the data, we then discuss the ability of using edge-on disks to determine the vertical structure of gas disks.

\section{Observations}

Imaging observations were performed with the Atacama Large $\mathrm{mm} / \mathrm{submm}$ Array (ALMA) in a moderately compact configuration. The project 2013.1.00387.S was observed on 17 and 18 May 2015 under excellent weather conditions. The correlator was configured to deliver very high spectral resolution with a channel spacing of $15 \mathrm{kHz}$ (and an effective velocity resolution of $40 \mathrm{~m} \mathrm{~s}^{-1}$ ). We observed simultaneously CO $J=2-1$, all the most intense hyperfine components of the CN $N=2-1$ transition, and the CS $J=5-4$ line.

Data was calibrated via the standard ALMA calibration script in the CASA software package (Version 4.2.2). Titan was used as a flux calibrator. The calibrated data was regridded in velocity to the Local Standard of Rest (LSR) frame using the "cvel" task, and exported through UVFITS format to the GILDAS package for imaging and data analysis. Atmospheric phase errors were small, providing high dynamic range continuum images and thermal noise limited spectral line data. The total continuum flux is $35 \mathrm{mJy}$ at $242 \mathrm{GHz}$ (with about $7 \%$ calibration uncertainty). With robust weighting, the $u v$ coverage provided by the $\sim 34$ antennas yields a nearly circular beam size close to $0.5^{\prime \prime}$. The CS images were produced at an effective spectral resolution of $0.1 \mathrm{~km} \mathrm{~s}^{-1}$; the rms noise is $3 \mathrm{mJy} / \mathrm{beam}$, i.e., about $0.27 \mathrm{~K}$ given the beam size of $0.48^{\prime \prime} \times 0.46^{\prime \prime}$ at PA $53^{\circ}$. For $\mathrm{CO}$, a spectral resolution of $0.08 \mathrm{~km} \mathrm{~s}^{-1}$ was used, and the rms noise is $4 \mathrm{mJy} /$ beam, i.e., about $0.37 \mathrm{~K}$ given the beam size of $0.51^{\prime \prime} \times 0.48^{\prime \prime}$ at PA $54^{\circ}$.

Figures 1 and 2 present the $\mathrm{CO}$ and CS channel maps, respectively, spectrally smoothed to $0.4 \mathrm{~km} \mathrm{~s}^{-1}$ for clarity. Figure 3 is a summary of the emission from $\mathrm{CO}, \mathrm{CS}$, and the dust continuum.

In addition, a CO $J=2-1$ spectrum of the clouds along the line of sight was obtained with the IRAM $30 \mathrm{~m}$ telescope, as described in Guilloteau et al. (2016).

\section{Results}

The CO $J=2-1$ and continuum results were partially reported by Guilloteau et al. (2016), who used them to measure the dust temperature.

\subsection{Images}

Figure 3 clearly shows that the disk is viewed close to edge-on. It also reveals a vertical stratification of the dust and molecules.

The modeling of the continuum image by Guilloteau et al. (2016) shows that the (millimeter) dust grains has a scale height of $12.7 \pm 0.3$ au at $100 \mathrm{au}$, increasing with a $0.34 \pm 0.04$ exponent. For comparison, the modeling of the near-infrared images by Grosso et al. (2003) shows that the (micron) dust grains have a larger scale height of $22.5 \pm 1.5$ au at 100 au when adopting the same definition and distance, and increasing more rapidly with a 1.25 exponent. Therefore, there is a clear indication of dust settling in this disk, with large grains preferentially close to the disk mid-plane.

The integrated intensity maps (Fig. 3) show that CS is significantly more confined towards the disk mid-plane than CO. As mentioned in Guilloteau et al. (2016), CO is contaminated by background emission from extended molecular clouds at four different velocities, which affects the derivation of the integrated emission and results in apparent asymmetries. The CS molecular emission extends up to radius of at least $300 \mathrm{au}$, and slightly more $(\sim 330 \mathrm{au})$ for $\mathrm{CO}$. On the contrary, the dust emission is confined within $200 \mathrm{au}$. The apparent distributions may be more a result of temperature gradients, excitation conditions, 
A. Dutrey et al.: Radial and vertical structure of the Flying Saucer

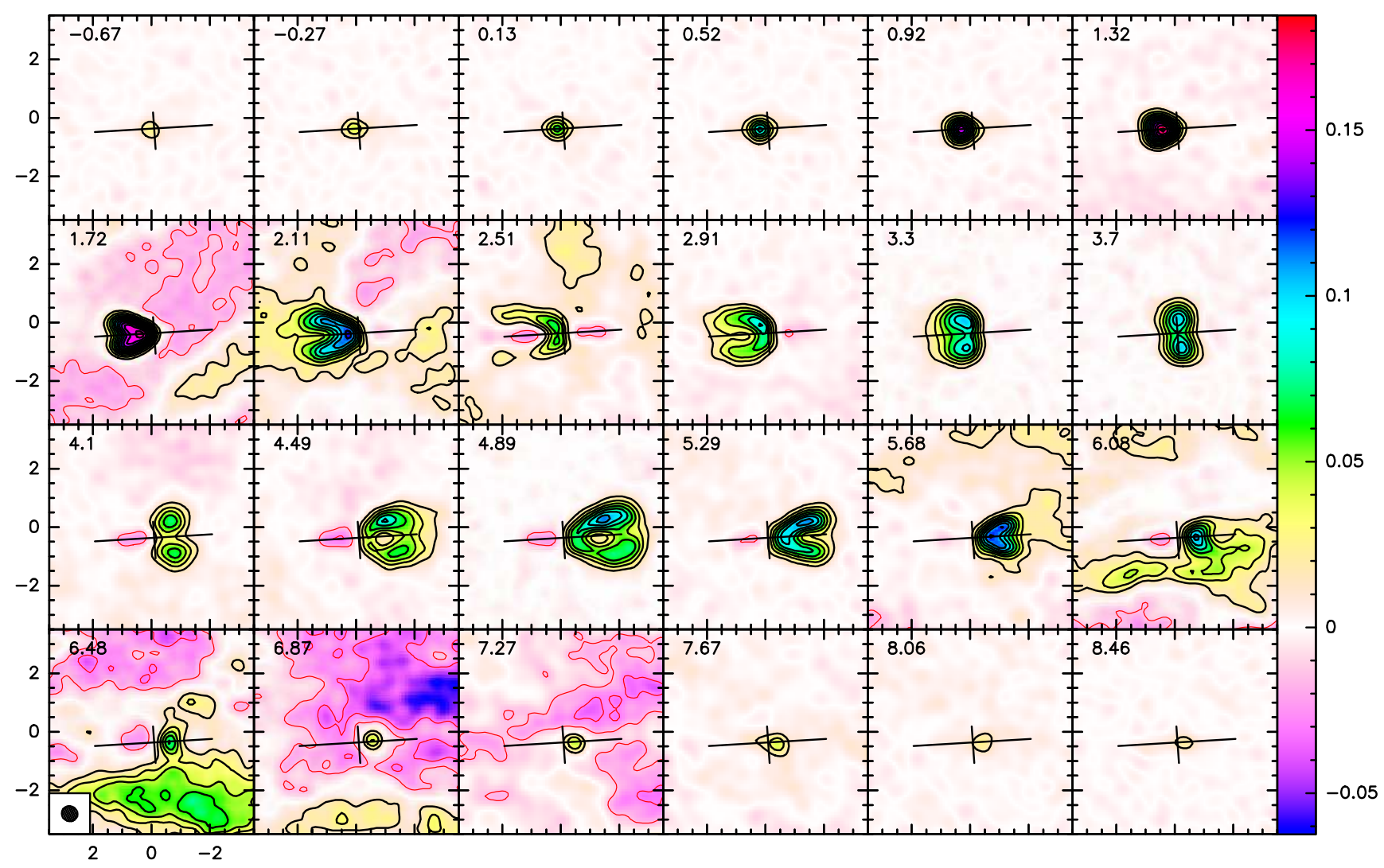

Fig. 1. Channel maps of the ${ }^{12} \mathrm{CO} J=2-1$ line emission toward the Flying Saucer. Contours are in steps of $15 \mathrm{mJy} /$ beam (1.22 K, approximately $7 \sigma)$ : negative contours are in red, positive contours in black, zero level contour omitted, and the color scale is given on the right. The cross indicates the position and orientation of the dust disk. The LSR velocity (in $\mathrm{km} \mathrm{s}^{-1}$ ) is indicated in the upper left corner of each panel.

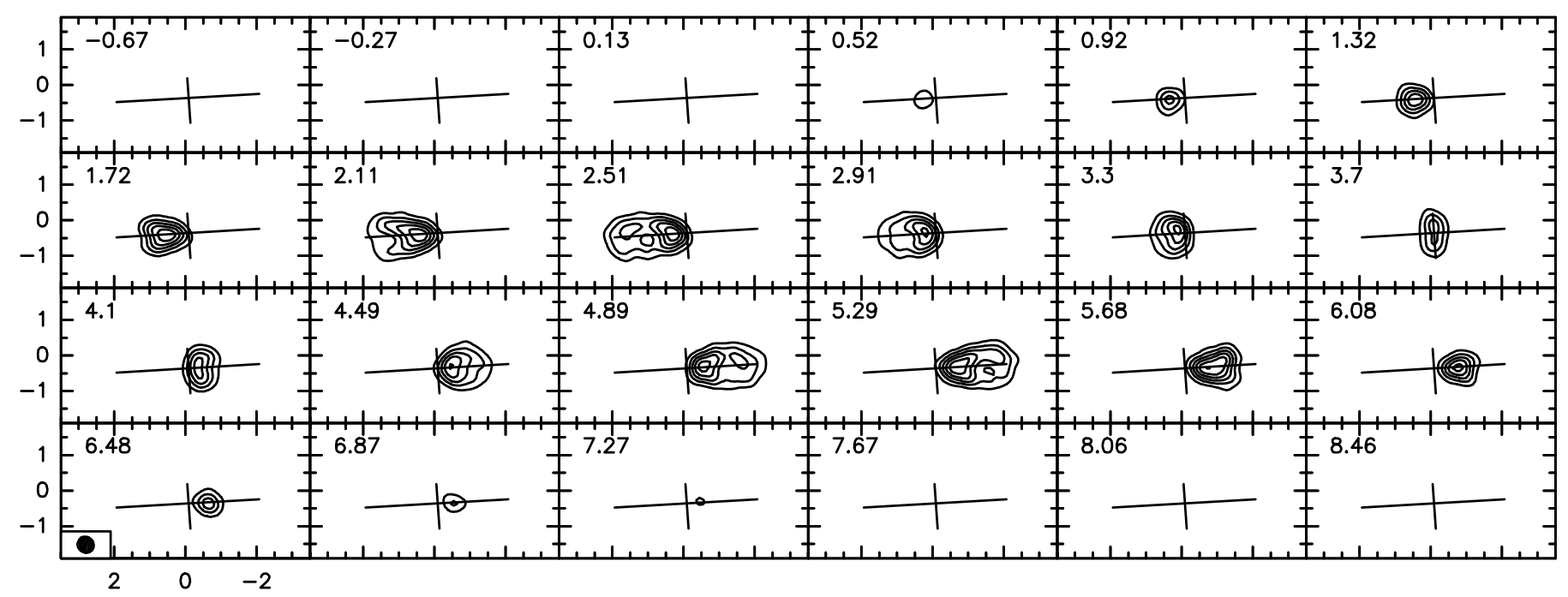

Fig. 2. As in Fig.1, but for the CS $J=5-4$ line. Contours are in steps of $15 \mathrm{mJy} /$ beam $(1.47 \mathrm{~K}$, approximately $9 \sigma)$.

and line opacities than of different abundance gradients for these molecules. The $\mathrm{CO} J=2-1$ line is much more optically thick than the CS line, and thus more sensitive to the (warmer) less dense gas high above the disk mid-plane. Along the mid-plane, self-absorption by colder, more distant gas, can result in lower apparent brightness. However, at the disk edges this effect should be small, so the higher brightness above the disk plane likely indicates a vertical temperature gradient with warmer gas above the plane.
The aspect of the iso-velocity contours (Fig. 3) is exactly what is expected from a Keplerian flared disk seen edge-on. In such a configuration, at an altitude $z$ above the disk plane, the disk only extends inwards to an inner radius depending on $z / H$, where $H$ is the scale height, so that the maximum velocity reached at altitude $z$ is limited by this inner radius. Thus the mean velocity decreases from the mid-plane to higher altitude, resulting in a "butterfly" shape of the iso-velocity contours. The effect is less pronounced for $\mathrm{CO}$, however, as its high optical depth 

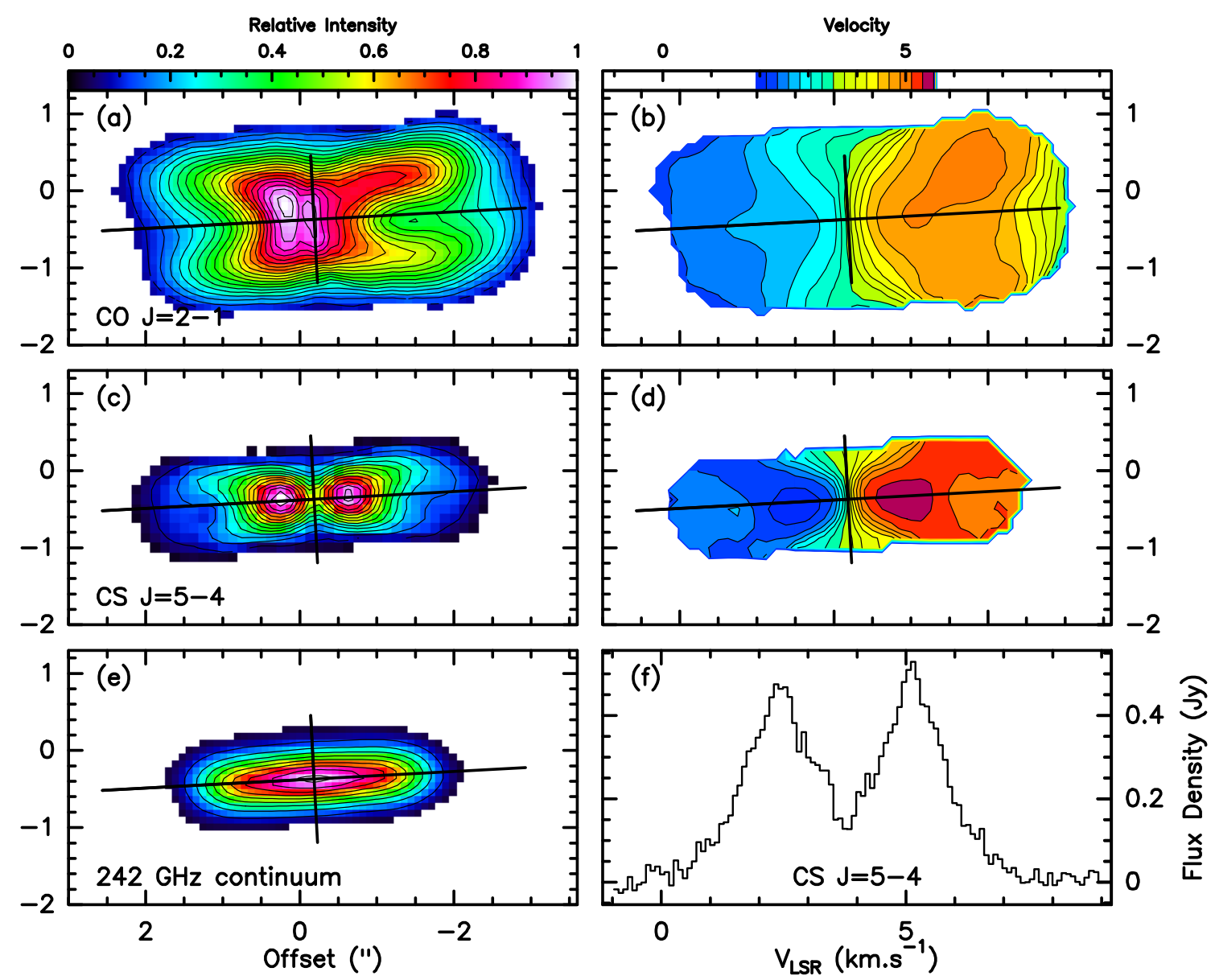

Fig. 3. Summary of the observations for CO, CS, and dust. CO $J=2-1$ integrated intensity map $(a)$ and isovelocity contours $(b)$, CS $J=5-4$ integrated intensity map $(c)$ and isovelocity contours $(d)$, continuum image at $242 \mathrm{GHz}(e)$ and $\mathrm{CS} J=5-4$ integrated spectrum $(f)$.

allows us to trace the emission well above the disk plane (isovelocity contours would be parallel for a cylindric distribution).

\subsection{Simple determination of the disk parameters}

To constrain the basic parameters of the disk, we made a simple model of the CS $J=5-4$ emission with DiskFit (Piétu et al. 2007) assuming power laws for the CS surface density $\left(\Sigma_{\mathrm{CS}}(r)=\right.$ $\left.\Sigma_{0}(r / 100 \mathrm{au})^{-p}\right)$ and temperature $T_{\mathrm{ex}}(r)=T_{0}(r / 100 \mathrm{au})^{-q}$. The disk was assumed to have a sharp outer edge at $R_{\text {out }}$. The vertical density profile was assumed Gaussian (see Eq. (1) Piétu et al. 2007), with the scale height a (free) power law of radius $h(r)=$ $H_{0}(r / 100 \mathrm{au})^{-h}$. The line emission was computed assuming a (total) local line width $\mathrm{d} V$ independent of the radius and LTE (i.e., $T_{0}$ represent the rotation temperature of the level population distribution).

In addition to the above intrinsic parameters, the model also includes geometric parameters: the source position $x_{0}, y_{0}$; the inclination $i$ and the position angle of the rotation axis PA; and the source systemic velocity $V_{\mathrm{LSR}}$ relative to the LSR frame.

Results are given in Table 1. This simple model allows us to determine the overall disk orientation, the systemic velocity, and the stellar mass, and gives an idea of the temperature required to provide sufficient emission. The apparent scale height $H_{0}$ derived at 100 au corresponds to a temperature of $53 \mathrm{~K}$, much larger than $T_{0}$. The difference may indicate that CS is substantially subthermally excited or, more likely, that CS emission only originates from above one hydrostatic scale height.
Table 1. CS disk modeling results.

\begin{tabular}{lcll}
\hline \hline Parameter & Value $($ at $100 \mathrm{au})$ & Unit & \\
\hline PA & $3.6 \pm 0.4$ & $\circ$ & PA of disk rotation axis \\
$i$ & $85.4 \pm 0.5$ & $\circ$ & Inclination \\
$V_{\mathrm{LSR}}$ & $3.755 \pm 0.003$ & $\mathrm{~km} \mathrm{~s}^{-1}$ & Systemic velocity \\
$M_{*}$ & $0.58 \pm 0.01$ & $M_{\odot}$ & Star mass $^{a}$ \\
$R_{\text {out }}$ & $290 \pm 7$ & $\mathrm{au}$ & Outer radius \\
$\mathrm{d} V$ & $0.17 \pm 0.01$ & $\mathrm{~km} \mathrm{~s}^{-1}$ & Local line width $^{b}$ \\
$\Sigma_{0}$ & $4.3 \times 10^{13} \pm 0.3 \times 10^{13}$ & $\mathrm{~cm}^{-2}$ & CS surface density \\
$p$ & $2.71 \pm 0.03$ & & Surface density exponent \\
$T_{0}$ & $18.0 \pm 0.5$ & $\mathrm{~K}$ & CS temperature \\
$q$ & $-0.18 \pm 0.03$ & & temperature exponent \\
$H_{0}$ & $25.8 \pm 0.3$ & $\mathrm{au}$ & Scale height of CS \\
$h$ & $-1.40 \pm 0.03$ & & Exponent of scale height \\
\hline
\end{tabular}

Notes. ${ }^{(a)}$ Assuming Keplerian rotation. ${ }^{(b)}$ Assumed constant with $r$. Errors are formal error bars from the fit. ${ }^{(c)}$ Apparent scale height (see Sect. 3.2).

\subsection{Position-velocity diagrams}

A more detailed understanding of the disk properties can be derived from the position-velocity (PV) diagram shown in Figs. 4 and 5 where several altitudes $z$ are shown. In these diagrams, radial straight lines (i.e., lines with $v(x)-V_{\text {disk }} \propto x$, where $x$ is the impact parameter, $v$ the velocity, and $V_{\text {disk }}$ the disk systemic velocity) trace a constant radius $r$ (see Appendix A for details). The blue straight lines indicate the outer radius $\left(R_{\text {out }} \simeq 330 \mathrm{au}\right)$. The blue curve is the Keplerian rotation curve $\sqrt{G M_{*} / r}$, with 
A. Dutrey et al.: Radial and vertical structure of the Flying Saucer
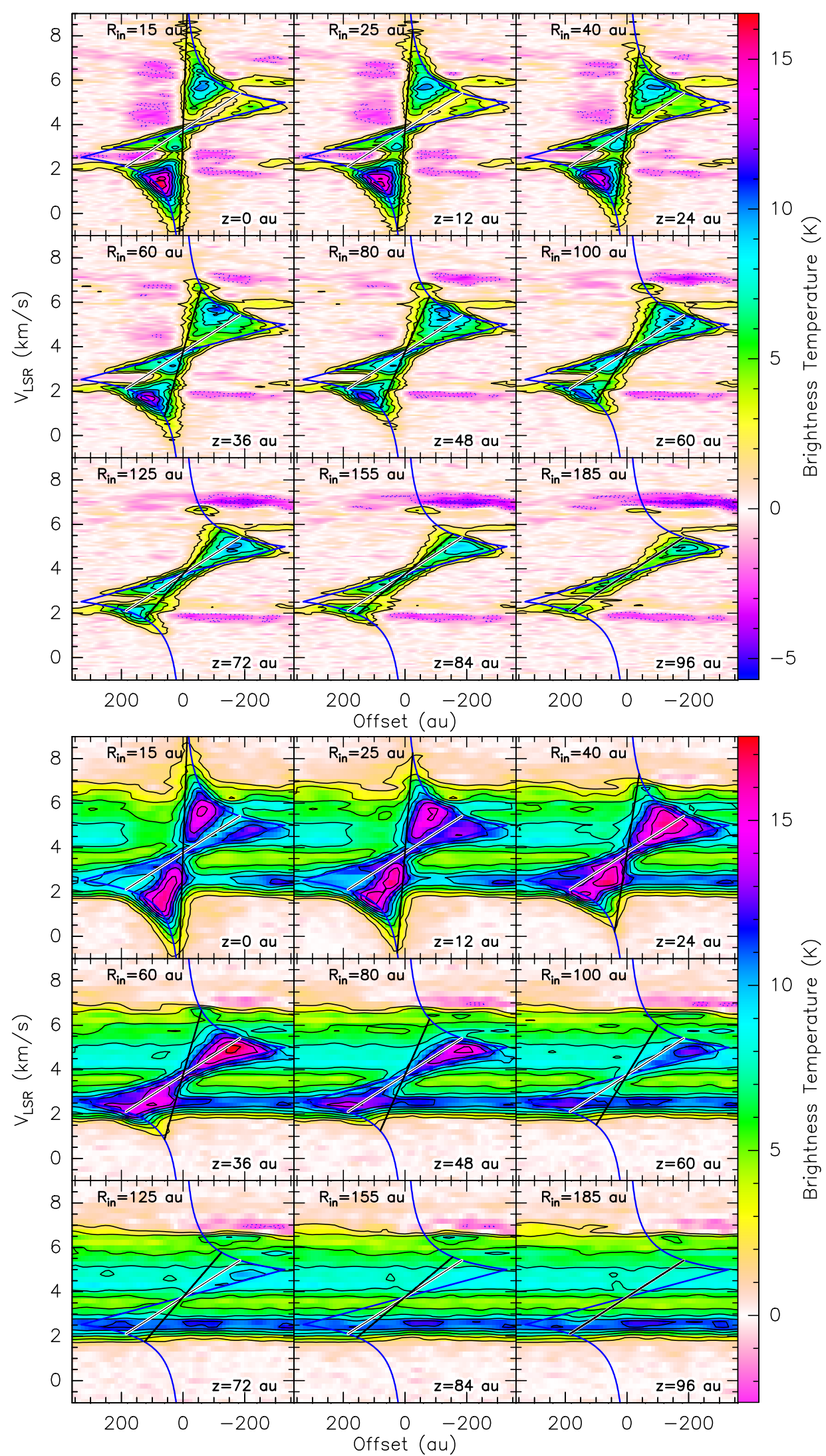

Fig. 4. Position-velocity diagram for CO $J=2-1$. Each panel is a cut at a different height above the plane (indicated in the lower right corner of each panel). The spatial resolution is $56 \mathrm{au}$. Top: without the background added (spectral resolution $0.08 \mathrm{~km} \mathrm{~s}^{-1}$ ). Bottom: with the background spectrum added. In each panel, the black line highlights the apparent inner radius whose value is quoted in the upper left corner. Here, the spectral resolution is $0.27 \mathrm{~km} \mathrm{~s}^{-1}$. 


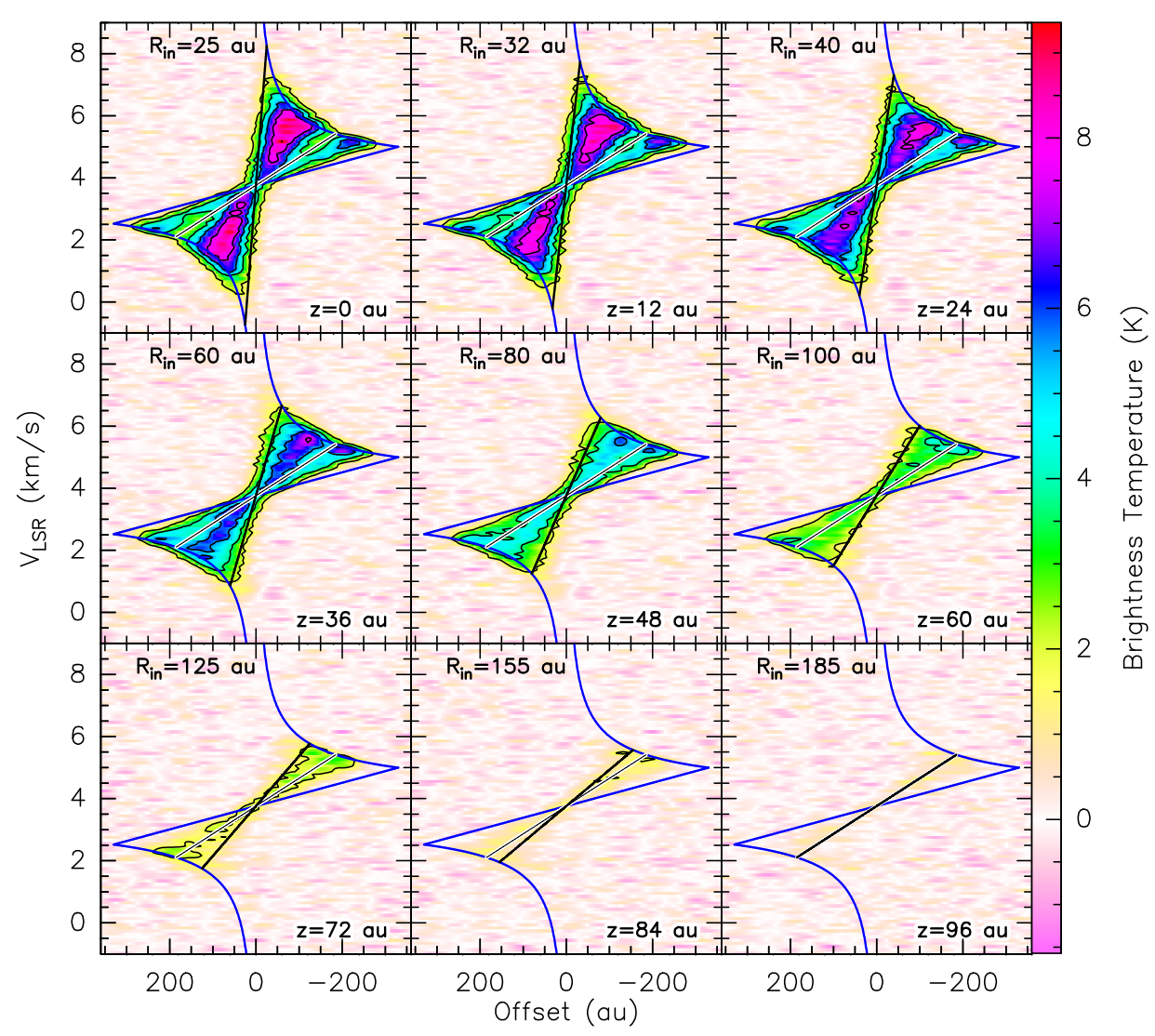

Fig. 5. As in Fig. 4, but for CS $J=5-4$ (spectral resolution $0.10 \mathrm{~km} \mathrm{~s}^{-1}$ ).

a stellar mass of $0.57 M_{\odot}$. The black line is the apparent inner radius $R_{\text {in }}$, and white-over-black line delineates the radius $r_{\text {dip }} \simeq 185 \mathrm{au}$ where a dip in emission is observed at low altitudes both in $\mathrm{CO}$ and in $\mathrm{CS}$.

The CO $J=2-1$ line provides a direct view into the thermal structure of the disk. As is true for the continuum emission, the background provided by the four extended molecular clouds identified in the $30 \mathrm{~m}$ spectrum modulates the apparent brightness of the disk since the ALMA array only measures the difference in emission between the disk and the background clouds. Because the $\mathrm{CO} J=2-1$ line is essentially optically thick in disks, we can simply recover a corrected CO PV diagram by adding the background spectrum obtained with the IRAM $30 \mathrm{~m}$ (see Guilloteau et al. 2016, their Fig. 1) to the observed $\mathrm{CO}$ emission, at least within the disk boundaries (in position and velocity). The result is given in Fig. 4 (bottom).

In the disk plane, while $\mathrm{CO}$ appears to extend down to very small radii $(<15 \mathrm{au}), \mathrm{CS}$ may have an inner radius around $25 \mathrm{au}$.

At a given location, the impact of the finite beam size depends on the brightness temperature gradients, which are different for CO and CS (because of different opacities and excitation conditions), therefore leading to different apparent structures. Nevertheless, all apparent inner radii increase with height above the disk plane, as described before for the analysis of iso-velocity curves. This happens because the disk is flaring due to the hydrostatic equilibrium, hence its vertical thickness is increasing with radius.

The disk mid-plane is also clearly colder than the brightest background molecular cloud at $2.8 \mathrm{~km} \mathrm{~s}^{-1}$, which has $J_{v}(T)=$ $11 \mathrm{~K}$, beyond about 100 au radius, being almost as warm as the second brightest cloud at $4.2 \mathrm{~km} \mathrm{~s}^{-1}$, with $J_{v}(T)=8 \mathrm{~K}$, at radii around $185 \mathrm{au}$. From this simple consideration, we safely constrain the mean disk mid-plane temperature, averaged over one beam, to be $13 \mathrm{~K}$ near 180 au after proper conversion of the brightness temperature outside of the Rayleigh-Jeans domain. It would rise to about $18 \mathrm{~K}$ at $100 \mathrm{au}$.

The PV diagrams above the disk plane indicate a warmer temperature in the upper layers, since no absorption is visible for $z>40$ au or so. Because of our limited linear resolution (about $56 \mathrm{au}$ ), with such a vertical temperature gradient the CO PV diagram only gives an upper limit to the disk mid-plane temperature because the scale height (about 10 au at $100 \mathrm{au}$ ) is substantially smaller than the linear resolution except at the disk edge.

We also note that CS and CO both show a drop in the emission intensity at radius $r_{\text {dip }} \simeq 185 \mathrm{au}$. This drop is somewhat more difficult to identify in the CO PV diagrams because of the background clouds. In $\mathrm{CO}$, the emission drop seems to disappear at a height of $40 \mathrm{au}$, suggesting it occurs only below about $30-40$ au given the limited angular resolution. Beyond a radius of $220 \mathrm{au}, \mathrm{CO}$ emission is observed again. In CS, the deficit of emission near 185 au extends somewhat higher, up to a height of $60 \mathrm{au}$.

Figure 6 also reveals a north-south asymmetry, the north side being brighter in $\mathrm{CS}$ and in $\mathrm{CO}$.

\section{New method of analysis}

\subsection{Deriving the brightness distribution from the position-velocity diagram}

For a homogeneous medium, the measured brightness temperature $T_{\mathrm{b}}$ is given by

$T_{\mathrm{b}}=(1-\exp (-\tau))\left(J_{v}(T)-J_{v}\left(T_{\mathrm{bg}}\right)\right)$ 

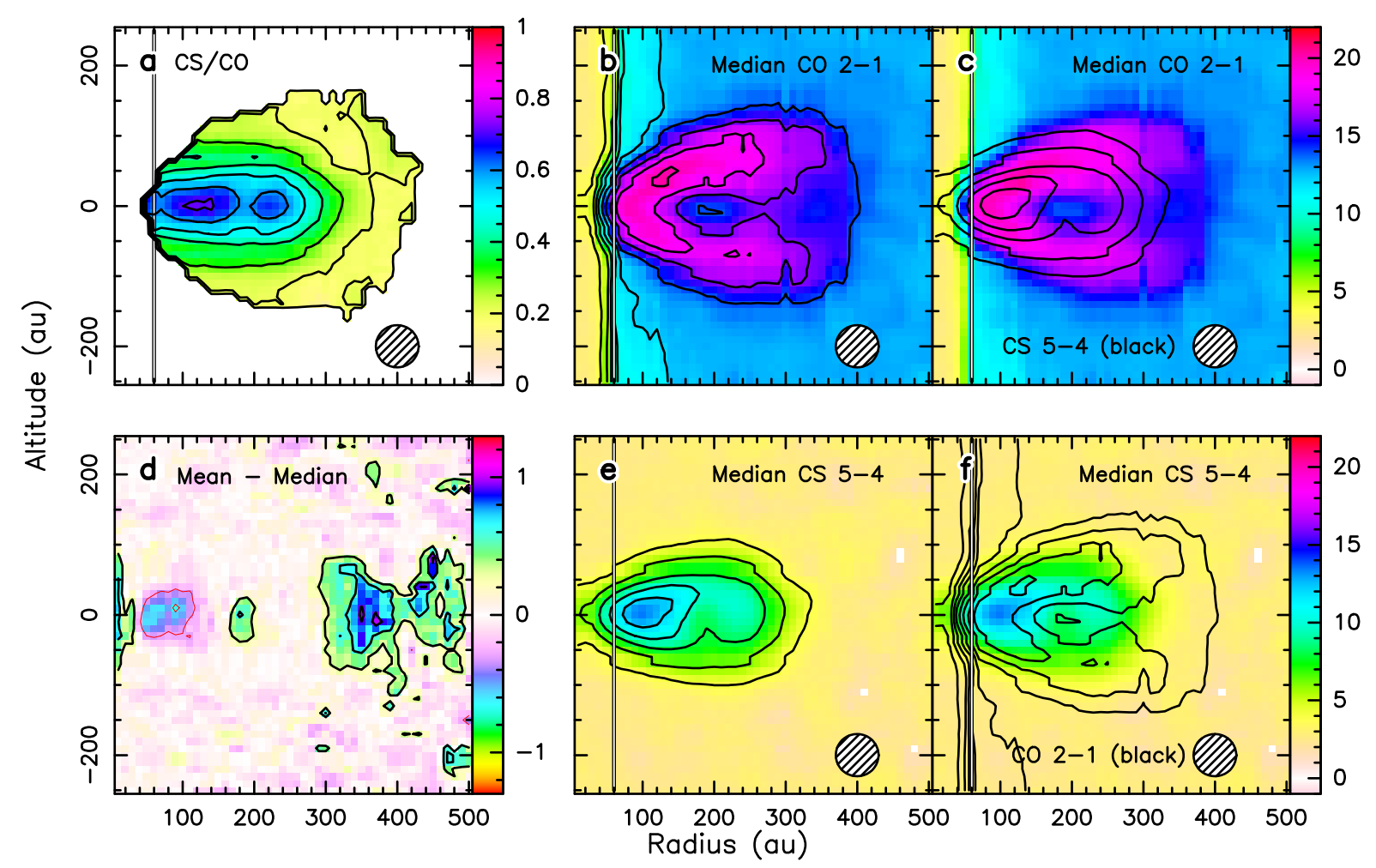

Fig. 6. Derivations of the gas temperature from the PV diagram. a) Ratio of the median TRD derived from CS over the median TRD derived from $\mathrm{CO}$ (contours are 0.1 to 1 by 0.1 ); b) CO median TRD, with contours from 4 to $22 \mathrm{~K}$ by spacing of $2 \mathrm{~K} ; c$ ) CO median TRD with contours of the CS median TRD overlaid; $d$ ) difference between the CS mean temperature and the CS median TRD (contours are $-0.6,-0.3,0.3$ and $0.6 \mathrm{~K}$ ); $e$ ) median CS TRD with contours from 4 to $12 \mathrm{~K}$ by spacing of $2 \mathrm{~K} ; f$ ) CS median TRD with contours of the CO median TRD. The spatial resolution is indicated. The vertical line delineates the area suffering from beam dilution.

where $J_{v}$ is the Planck function multiplied by $c^{2} / 2 k v^{2}$,

$$
J_{v}(T)=\frac{h v}{k} \frac{1}{\exp (h v /(k T))-1} .
$$

Since in a PV diagram a radial line represents a constant radius, we can recover the disk temperature from the thermalized and optically thick CO $J=2-1$ transition by averaging the observed brightness along such radial lines in regions where the signal is sufficiently resolved spectrally and spatially. This averaging process yields the mean radiation temperature, $J_{v}(T)-J_{v}\left(T_{\mathrm{bg}}\right)$, from which the temperature $T$ is derived. The disk being seen edge-on, cuts at various altitudes $z$ provide a direct visualization of the gas temperature versus radius $T(r, z)$, although only at the angular resolution of the observations. The $2 \mathrm{D}$ image resulting from the application of this averaging process is called hereafter the tomographically reconstructed distribution (TRD).

While the CO $J=2-1$ TRD is just the temperature distribution, for optically thinner or non-thermalized lines the interpretation is more complex because the TRD is a function of both the temperature and local density. Nevertheless, it also provides a direct measurement of the altitude of the molecular layer.

We use the PV diagrams of CO and CS transitions to derive their respective TRDs and show them in Fig. 6. The derivations were performed on data cubes without continuum subtraction because the subtraction, which was needed to compute the iso-velocity contours, may lead to substantial problems near the peak of the continuum. For $\mathrm{CO}$, which is optically thick, the $\operatorname{TRD}(r, z)$ map is obtained using the PV diagram with the CO emission from the background clouds added since that emission is fully resolved out by the ALMA observations. This explains why the derived $\mathrm{CO}$ temperature brightness is on the order of
$15 \mathrm{~K}$ around the disk, in agreement with the values derived from the $\mathrm{CO}$ spectrum taken with the IRAM $30 \mathrm{~m}$ radio telescope (Guilloteau et al. 2016).

In each case, we calculated a map of the mean, median, and maximum brightness along each radius. For $\mathrm{CO}$, the mean and maximum brightness are contaminated by the background clouds, and the median is expected to be a better estimator. Furthermore, for CS, we found that the mean and the median always give results that differ by less than $1 \mathrm{~K}$ inside the disk at $r<350$ au (see Fig. 6, panel (d), which shows the difference between the mean and the median for CS 5-4). This indicates that the derivations are robust and do not suffer from significant biases. The observed TRDs clearly confirm the location of the molecular layer above the mid-plane, but the CS emission peaks at a lower temperature and is located below the $\mathrm{CO}$ emission. The ratio of the CS TRD over the CO TRD confirms these behaviors (see Fig. 6, panel (a)).

The Keplerian shear implies that the spatial averaging of the derived brightness is not the same everywhere inside the disk. Indeed, at a radius $r$ corresponding to a velocity $v(r)$, the smearing due to the Keplerian shear is given by $\mathrm{d} r=2 r \mathrm{~d} v / v(r)$, where $\mathrm{d} v$ is the local linewidth, which is due to a combination of thermal and turbulent broadening. This limits the radial resolution which can be obtained in the disk outer parts. For instance, for $\mathrm{CO}$, at $20 \mathrm{au}$, assuming a temperature of $30 \mathrm{~K}$ and a turbulent broadening $\left(0.05 \mathrm{~km} \mathrm{~s}^{-1}\right)$ similar to that observed in the TW Hydrae disk by Teague et al. (2016), the $\mathrm{d} r$ would be on the order of $\sim 2 \mathrm{au}$, a value that would not affect studies at spatial resolution down to $0.1^{\prime \prime}(12 \mathrm{au})$ or so. On the contrary, at radius $200 \mathrm{au}$, the $\mathrm{d} r$ would be on the order of 35 au for the same line width, dropping to 20 au assuming the same turbulence but a (mid-plane) 
temperature of $7 \mathrm{~K}$. This limits the gain obtained with high angular resolution only at large radii. It also explains why the apparent extent of the tomographically reconstructed distribution in Fig. 6 exceeds the disk outer radius more than expected from the angular resolution only.

Nevertheless, this smearing is purely radial and the vertical structure is not affected; the smearing is the same above and below the mid-plane at a given radius (assuming there is no vertical temperature gradient). This occurs because the disk is edge-on, but it is no longer true in less inclined disks. In such disks, the smearing resulting from the local line width will limit the effective resolution radially and vertically.

Finally, we note that this direct method of analysis, specific to edge-on disks, complements the classical channel maps studies by providing a more synthetic but direct view of the vertical disk structure.

\subsection{CO modeling using DiskFit}

To go beyond the resolution-limited information provided in Fig. 6, we study here the impact of several key parameters of the disk by performing grids of models to better constrain the disk geometry and structure. We use the ray-tracing model DiskFit (see Sect. 3.2, Piétu et al. 2007). For simplicity, we assume LTE conditions, which is appropriate for CO. Here the temperature structure is more complex than the simple vertically isothermal model described in Sect. 3.2. The atmosphere temperature is given by

$T_{\mathrm{atm}}(r)=T_{\mathrm{atm}}^{0}\left(\frac{r}{r_{0}}\right)^{-q_{\mathrm{atm}}}$

and the mid-plane temperature is given by

$$
T_{\text {mid }}(r)=\min \left(T_{\text {atm }}(r), T_{\text {mid }}^{0}\left(\frac{r}{r_{0}}\right)^{-q_{\text {mid }}}\right)
$$

In between for an altitude of $z<z_{q}$, the temperature is defined by

$T(r)=\left(T_{\text {atm }}(r)-T_{\text {mid }}(r)\right)\left(\cos \left(\frac{\pi z}{2 z_{q} H(r)}\right)\right)^{2 \delta}+T_{\text {mid }}(r)$,

where $H(r)$ is the hydrostatic scale height (defined by $T_{\text {mid }}(r)$ ). Provided $q_{\text {atm }}>q_{\text {mid }}$, there is a radius $R_{q}$ beyond which the temperature becomes vertically isothermal. We note that for $q_{\mathrm{mid}}=0$, this definition is identical to that used by Dartois et al. (2003).

The models of the molecular emission were performed together with the continuum emission not subtracted. The continuum model uses, in particular for the density and temperature, the parameters defined in Table 1 of Guilloteau et al. (2016). We take into account the spatial resolution by convolving all models by a $0.5^{\prime \prime}$ circular beam. In all models, the outer radius $r_{\text {out }}$ is taken at $330 \mathrm{au}$, in agreement with the value derived from the PV diagram.

We find that a small departure from edge-on inclination by 2 3 degrees is sufficient to explain the small north-south brightness asymmetry visible in Fig. 6 . The most probable value is $i=87^{\circ}$ (see Sect. 5.1), a value used for all further models.

In a first series of models, we assume a $\mathrm{CO}$ vertical distribution that follows the $\mathrm{H}_{2}$ density distribution, i.e., assuming a constant abundance. For the $\mathrm{H}_{2}$ density distribution, we assume either power laws or exponentially tapered distribution following the prescription given in Guilloteau et al. (2011). We explored
CO surface densities ranging from $10^{16}$ to $10^{19} \mathrm{~cm}^{-2}$ at $100 \mathrm{au}$. To account for the observed brightness at high altitudes above the disk plane, we find that the $\mathrm{CO}$ surface density at $100 \mathrm{au}$ must be at least on the order of $5 \times 10^{17} \mathrm{~cm}^{-2}$, with a power law index on the order of $p=1.2$ for the radial distribution. We also explored the impact of the temperature distribution $z_{q}, \delta, T_{\mathrm{atm}}^{0}$, $q_{\mathrm{atm}}, T_{\mathrm{mid}}^{0}, q_{\mathrm{mid}}$, and $R_{q}$. Best runs are obtained for mid-plane temperatures $T_{\text {mid }} \approx 10 \mathrm{~K}$ at $100 \mathrm{au}, q_{\mathrm{mid}}=0.4$ leading to about $6 \mathrm{~K}$ at the outer disk radius, and $17 \mathrm{~K}$ at 26 au (CO snowline location), $T_{\mathrm{atm}}^{0}=50 \mathrm{~K}, q_{\mathrm{atm}}=0-0.2, \delta=2$, and $z_{q}=1.3-2$.

However, none of these models, which assume CO molecules are present everywhere in the disk, properly reproduces the $\mathrm{CO}$ depression observed around the mid-plane (see panel (b) of Fig. 6). This remains true even if the mid-plane temperature is set to values of 5-7 K, matching the temperature of large grains measured by Guilloteau et al. (2016).

We attempted to perform more realistic models by assuming complete molecular depletion (abundance $X_{\mathrm{p}}(\mathrm{CO})=0$ ) in the disk mid-plane. In this model, the zone where $\mathrm{CO}$ is present is delimited upwards by a depletion column density $\Sigma_{\text {dep }}$ and downwards by the $\mathrm{CO}$ condensation temperature. Molecules of $\mathrm{CO}$ are present (with a constant abundance $X_{\mathrm{u}}(\mathrm{CO})=10^{-4}$ ) when the $\mathrm{H}_{2}$ column density from the current $(r, z)$ point to the disk surface (i.e., towards $(r, \infty))$ exceeds a given threshold $\Sigma_{\text {dep }}$, reflecting the possible impact of photo-desorption of molecules, or when the temperature $T(r, z)$ is above $17 \mathrm{~K}$. Such a model takes into account the possible presence of $\mathrm{CO}$ in the inner disk midplane inside the $\mathrm{CO}$ snowline radius, and also at large radii because as soon as the surface density becomes low enough, $\mathrm{CO}$ emission can again be located on the mid-plane. In this model, the $\mathrm{CO}$ surface density radial profile $\Sigma(r) \times X_{\mathrm{u}}(\mathrm{CO})$ (where $\Sigma(r)$ is the $\mathrm{H}_{2}$ surface density profile) is constrained because there needs to be sufficient opacity for the CO $J=2-1$ at high altitudes above the disk mid-plane in order to reproduce the observed brightness. The derived $\mathrm{H}_{2}$ densities are then strictly inversely proportional to the assumed $X_{\mathrm{u}}(\mathrm{CO})$.

Table 2 gives the parameters of the best model we found with this approach. Unfortunately the current angular resolution of the data limits the analysis. Because of this limited angular resolution, parameters $T_{\mathrm{atm}}^{0}, \delta, z_{q}$ are strongly coupled; $z_{q}$ also depends implicitly on $T_{\text {mid }}$ because it is the number of hydrostatic scale heights at which the atmospheric temperature is reached. In practice, Table 2 only confirms a low mid-plane temperature $(10 \mathrm{~K}$ at $100 \mathrm{au}$ ), and temperatures at least a factor of 2 larger than this in the $\mathrm{CO}$ rich region, consistent with the (spatially averaged) values derived in Sect. 3.3. Only higher spatial resolution data would allow us to break the degeneracy and accurately determine the vertical temperature gradient (see Sect. 5.4).

Furthermore, even this model only qualitatively reproduces the brightness distribution of the $\mathrm{CO}$ emission around the midplane. In particular, the shape of the depletion zone is difficult to evaluate from the current data. We also fail to reproduce the rise of the $\mathrm{CO}$ brightness after $250 \mathrm{au}$, most likely because our model does not include an increase in the temperature in the outer part.

Most chemical models (e.g., Reboussin et al. 2015) predict that there is some $\mathrm{CO}$ at low abundance $\left(X_{\mathrm{p}}(\mathrm{CO}) \sim 10^{-8}-10^{-6}\right)$ in the mid-plane, depending on the grain sizes and the local dustto-gas ratio, contrary to our simple assumption of $X_{\mathrm{p}}(\mathrm{CO})=0$. Such low abundances would not impact our determination of the $\mathrm{CO}$ surface density, which only relies on the need to have sufficient optical thickness in the upper layers. However, $\mathrm{CO}$ could start being sufficiently optically thick around the mid-plane, diminishing the contrast between the mid-plane and the molecular layer. We estimated through modeling that this happens for 
Table 2. Model derived from the CO observations.

\begin{tabular}{llll}
\hline \hline Parameter & Value & Unit & Parameters at $100 \mathrm{au}$ \\
\hline$T_{\mathrm{atm}}^{0}$ & 50 & $\mathrm{~K}$ & Atmosphere temperature \\
$q_{\mathrm{atm}}$ & 0.4 & & \\
$z_{q}$ & 3 & & \\
$\delta$ & 2 & & \\
$T_{\text {mid }}^{0}$ & 10 & $\mathrm{~K}$ & Mid-plane temperature \\
$q_{\text {mid }}$ & 0.4 & & \\
$\Sigma_{0}$ & $10^{23}$ & $\mathrm{~cm}^{-2}$ & $\mathrm{H}_{2}$ surface density \\
$p$ & 0.5 & & Surface density parameter \\
$R_{\mathrm{C}}$ & 50 & $\mathrm{au}$ & Radius for exponential decay \\
$H_{0}$ & 11.3 & $\mathrm{au}$ & Scale height \\
$h$ & -1.3 & & Exponent of scale height \\
$R_{\text {out }}$ & 330 & $\mathrm{au}$ & Outer radius \\
$i$ & 87 & $\circ$ & Inclination \\
$\Sigma_{\text {dep }}$ & $10^{22}$ & $\mathrm{~cm}$ & Surface density for depletion \\
$T_{\text {dep }}$ & 17 & $\mathrm{~K}$ & Depletion temperature \\
$X_{\mathrm{u}}(\mathrm{CO})$ & $10^{-4}$ & & CO abundance in upper layers \\
$X_{\mathrm{p}}(\mathrm{CO})$ & 0 & & CO abundance in mid-plane \\
$M_{\text {disk }}$ & $0.7 \times 10^{-3}$ & $M_{\odot}$ & Disk mass \\
\hline
\end{tabular}

Notes. $\Sigma(r)$ corresponds to Eq. (5) in Guilloteau et al. (2011), $H(r)=$ $H_{0}(r / 100 \mathrm{au})^{-h}$, and $T(r)$ is defined in Eqs. (1)-(3), this paper. The disk is in Keplerian rotation around a $0.57 M_{\odot}$. Only the product of $\Sigma_{0} \times X_{\mathrm{u}}(\mathrm{CO})$ is constrained by the observations: changing $X_{\mathrm{u}}(\mathrm{CO})$ will change $\Sigma_{0}$ and $M_{\text {disk }}$ by the inverse amount.

$X_{\mathrm{p}}(\mathrm{CO}) \geq 3 \times 10^{-8}$. In such cases, the brightness distribution becomes similar to that of the undepleted case. Observations of a less abundant isotopolog would be a better probe of the midplane depletion.

We thus conclude that the current data are insufficient to distinguish between molecular depletion and a very cold mid-plane, but indicate a rise in mid-plane temperature beyond $200 \mathrm{au}$.

In Fig. 7, we overlay the brightness temperature derived from the observations to the structure of the model given in Table 2. The brightness temperature from the model is compared to the observations and is also shown in Fig. 8 at three different angular resolutions of $0.5^{\prime \prime}, 0.3^{\prime \prime}$, and $0.1^{\prime \prime}$.

\section{Discussion}

\subsection{Overall disk structure}

The analysis of the $\mathrm{CO}$ and $\mathrm{CS}$ brightness temperature patterns shows that there is no significant departure from a simple disk geometry at the linear resolution of $60 \mathrm{au}$, with the exception of the north-south brightness difference. This may be due to an intrinsic asymmetry; however, the same effect can also be produced if the disk is slightly inclined by a few degrees from edgeon. Owing to the flaring and radial temperature gradient, the optically thick emission of the far side always originates from slightly warmer gas (Guilloteau \& Dutrey 1998). Indeed, we find that an inclination angle of $87^{\circ}$ is enough to account for the north-south dissymmetry, with the southern part of the disk being closer to us. This inclination (and orientation) is in very good agreement with that of $86 \pm 1^{\circ}$ derived by Grosso et al. (2003) from near-infrared (NIR) observations. The brightness distributions of the NIR images also show that the southern part of the disk is closer to us. Finally, there is no apparent sign of warp beyond a radius of about $50 \mathrm{au}$.

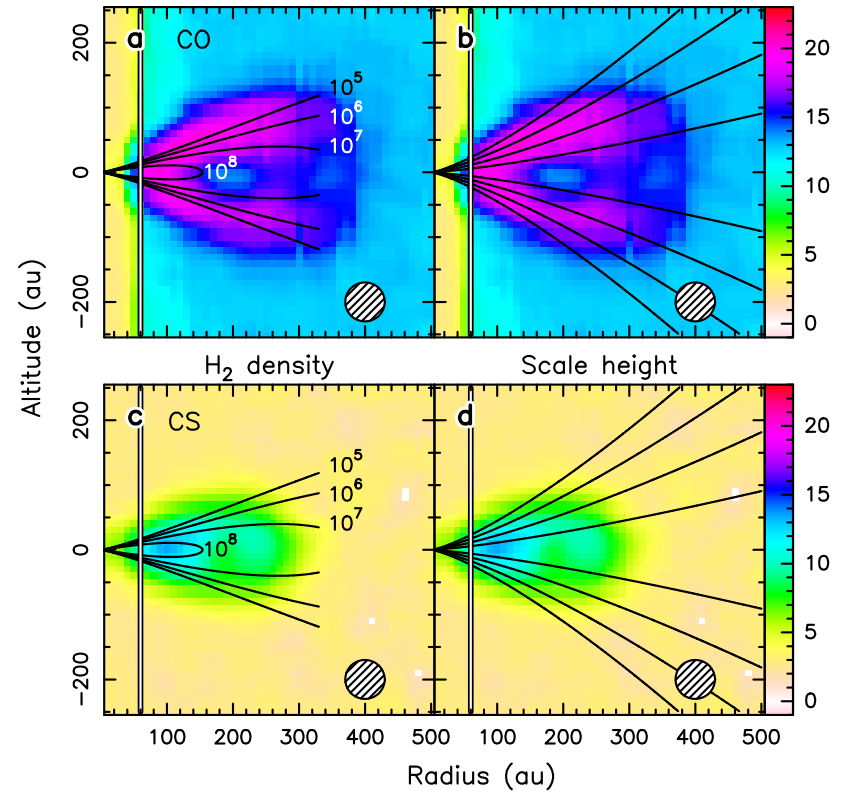

Fig. 7. Superimposition of the $\mathrm{CO}$ and CS median TRD to a standard disk model. a) CO TRD (color) and the $\mathrm{H}_{2}$ volume density superimposed in labeled contours from $10^{5}$ up to $10^{8} \mathrm{~cm}^{-3} ; b$ ) CO TRD with the scale height superimposed in black contours from 1 to 4 scale heights; panels $c$ and $d$ as panels $a$ and $b$, but for CS. The model corresponds to the parameters given in Table 2.

Figure 7 shows that the $\mathrm{CO}$ emission is above one scale height, while the CS emission appears slightly below and less extended vertically. This is not surprising because CS $J=5-4$ is excited only at the high densities near the mid-plane, while CO $J=2-1$ is easy to thermalize at densities as low as a few $10^{3} \mathrm{~cm}^{-3}$ (panels a-c of Fig. 7 trace the density distribution). As a consequence, the convolution by the $\sim 56$ au beam leads to different positions of the peak of the emission layer. The $\mathrm{CO}$ layer is vertically resolved and extended, while the CS emission is vertically unresolved and peaks just below one scale height. These vertical locations for the $\mathrm{CO}$ and $\mathrm{CS}$ layers are consistent with predictions by chemical models (see Dutrey et al. 2011, Fig. 8 where CS peaks between 1 and 2.5 scale heights). A CO layer above the mid-plane has already been observed in the disk of HD 163296 (de Gregorio-Monsalvo et al. 2013; Rosenfeld et al. 2013).

We also estimate for the first time the amplitude of the vertical temperature gradient between the cold mid-plane, the molecular layer, and the $\mathrm{CO}$ atmosphere at radii between 50 and $300 \mathrm{au}$. The gas vertical temperature gradient derived at $100 \mathrm{au}$ is in agreement with that predicted by thermo-chemical models (e.g., Cleeves et al. 2016) with a mid-plane at about 8-10 K and a temperature of $25 \mathrm{~K}$ reached at two scale heights. The main limitation here is the linear resolution of $60 \mathrm{au}$. Nevertheless, the observed pattern of the brightness distribution suggests the existence of warmer gas inside a radius of about 50-80 au. Any inner hole of radius $<15$ au cannot be seen in these data, due to our sensitivity limit.

\subsection{Radial profile of CO near the mid-plane}

We discuss here two possible origins for the emission drop in the CO radial profile.

Rise of the $\mathrm{CO}$ brightness a large radius: In the upper layers the $\mathrm{CO}$ brightness decreases smoothly with radius, while in the 
mid-plane we observe a rise in $\mathrm{CO}$ brightness beyond a radius of about $200 \mathrm{au}$. The transition radius coincides with the mmemitting dust disk outer boundary, $\sim 187$ au in the simple model from Guilloteau et al. (2016), while in scattered light the disk is nearly as extended as the $\mathrm{CO}$ emission (Grosso et al. 2003). This suggests that a change in grain size distribution may also play a role here. With the dust composed of mostly micron-sized grains better coupled with the gas (see Pontoppidan et al. 2007), more stellar light may be intercepted and may result in a more efficient heating of the gas, as suggested by recent chemical models (Cleeves 2016). Moreover, at the expected densities in the disk mid-plane, the dust and gas temperatures should be strongly coupled; a dust temperature rise is also naturally expected when the disk becomes optically thin to the incident radiation and for the re-emission, as shown by, e.g., D’Alessio et al. (1999).

Also, as the $\rho$ Oph region is bathed in a higher-than-average UV field due the presence of several B stars, this effect may be reinforced by additional heating due to a stronger ambient UV field. However, the Flying Saucer is located on the eastern side of the dark cloud, whose dense clouds absorb the UV from the B stars, located mainly on the western side. Figures 1a and $4 a$ in Lim et al. (2015) show that there is a clear dip in the far-UV emission due to the dark cloud silhouette. Therefore, at the location of the Flying Saucer $(l=353.3, b=16.5)$ the ambient far-UV field cannot be very large.

Apparent gap at radius $185 \mathrm{au}$ : The presence of an apparent gap at $\sim 185$ au in both $\mathrm{CO}$ and CS is puzzling. Its existence in $\mathrm{CO}$ indicates that a change in temperature and/or beam dilution is the primary cause for this deficit, as $\mathrm{CO}$ is easily thermalized and optically thick. This suggests that the disk mid-plane warms up beyond this radius, before the emission fades again near the disk edge (on the order of $\sim 330 \mathrm{au}$ ) where $\mathrm{CO}$ becomes optically thin and CS unexcited.

An alternative explanation is that it is the result of a real gap in the molecular distribution, smoothed out by our limited angular resolution. The optically thick $\mathrm{CO}$ line traces material at low and at high altitude (up to 3-4 scale heights above the midplane), while CS $J=5-4$, a high density tracer, is more optically thin and is only observed in high density regions (at typically one scale height). Contrary to a face-on or inclined disk, the gap can be seen in ${ }^{12} \mathrm{CO}$ because the disk is edge-on.

The brightness minimum at 185 au is only seen at low altitudes, typically between the mid-plane and an altitude of 40 au in $\mathrm{CO}$, and slightly higher in $\mathrm{CS}$, so the putative gap cannot extend upwards in the disk. This morphology remains consistent with expectations for gaps created by planets, provided the Hills radius is smaller than the disk scale height.

\subsection{Deriving the gas density from CS excitation conditions}

In CS $J=5-4$, we observe a similar north-south asymmetry to that in $\mathrm{CO} J=2-1$. This indicates that the emission has a substantial optical thickness along the line of sight.

The brightness ratio of $\mathrm{CS}$ over $\mathrm{CO}$ is within the range $0.5-$ 0.7 until a radius of about 250 au and drops quickly beyond. As indicated by the iso-density contours shown in Fig. 7, this is consistent with the decreasing $\mathrm{H}_{2}$ density with radius because the CS $J=5-4$ transition is thermalized at a few $10^{6} \mathrm{~cm}^{-3}$ (Denis-Alpizar et al., in prep.).

On the contrary, the observed ratio of $0.5-0.7$ in the inner disk mid-plane cannot be explained by excitation conditions. A simple escape probability calculation using the $\mathrm{CO}$ freeze out temperature $T_{k}=17 \mathrm{~K}$ (which is in agreement with the CS temperature derived from the simple analysis), a surface density of $4 \times 10^{13} \mathrm{~cm}^{-2}$, and a local line width of $0.3 \mathrm{~km} \mathrm{~s}^{-1}$ (obtained from a simple analysis with DiskFit using a power law surface density distribution) yields $T_{\text {ex }}=12 \mathrm{~K}$ for a density of $10^{6} \mathrm{~cm}^{-3}$. This density is much lower than expected in the disk given the dust emission observed at mm wavelengths, and lower than the values derived from our $\mathrm{CO}$ modeling.

The ratio is better explained as resulting from different beam dilutions in CO and CS. At $200 \mathrm{au}$, the CO emission is spatially resolved, while the region emitting in CS $J=5-4$ must fill only $50 \%$ of the synthesized beam, i.e., must have a thickness of only $\sim 30$ au. The emission peak being located $\sim 45$ au above the plane at this radius, the CS emitting layer must be confined between about 30 and 60 au there. Furthermore, the density at $r=200$ au and $z=60$ au is about $10^{6} \mathrm{~cm}^{-3}$ in our fiducial disk model, so that the upper layers are no longer dense enough to excite the $J=5-4$ line. Hence the observed $\mathrm{CS} / \mathrm{CO}$ brightness ratio is roughly consistent with a molecular layer extending above one scale height, with subthermal excitation of the CS $J=5-4$ transition truncating the CS brightness distribution upwards.

In this interpretation, the CS layer is expected to be thinner for higher $J$ transitions. An angular resolution around $0.2^{\prime \prime}$ would be needed to resolve the CS layer.

At smaller radii, the $\mathrm{CO}$ and $\mathrm{CS}$ layer both become unresolved vertically, but the ratio of their respective thickness is not expected to change significantly, leading to a nearly constant brightness ratio.

\subsection{Limits: angular resolution, local line width, and inclinations}

Figure 8 shows the TRD of the model obtained with DiskFit assuming $\mathrm{CO}$ depletion around the mid-plane at three different angular resolutions, $0.5^{\prime \prime}, 0.3^{\prime \prime}$, and $0.1^{\prime \prime}$. The impact of the resolution on this apparent brightness distribution is striking. For comparison, the intrinsic temperature $T(r, z)$ distribution is shown in panel $(\mathrm{g})$ for all points where the $\mathrm{H}_{2}$ density exceeds $10^{4} \mathrm{~cm}^{-3}$. Panels (f) and (g) show the impact of the small Keplerian shear compared to the local line width at the disk edge (which spreads the TRD beyond the outer disk radius, see Sect. 4.1), and of the small deviation from a pure edge-on disk (which results in a top/bottom asymmetry). For this specific disk structure, the peak brightness is lowered by a factor of 2 when degrading the angular resolution from $0.1^{\prime \prime}$ to $0.5^{\prime \prime}$. The inner $\mathrm{CO}$ disk, the radius of the $\mathrm{CO}$ snowline, and the whole gas distribution can be resolved at $0.1^{\prime \prime}$, but not at $0.5^{\prime \prime}$. The stratification of the molecular layer can only be studied at $0.1^{\prime \prime}$ resolution, down to about 30 au where the scale height becomes too small compared to the linear resolution for a direct measurement. We also observe a displacement of the peak brightness towards larger radii at $0.5^{\prime \prime}$ resolution compared to its (true) location at $0.1^{\prime \prime}$ resolution, which occurs because the vertical extent of the $\mathrm{CO}$ emission at large radii is larger, due to the flaring. Finally, at $0.5^{\prime \prime}$ we find that it is not possible to determine the shape of the (partly) depleted zone around the mid-plane beyond the $\mathrm{CO}$ snowline radius. Even a resolution of $0.3^{\prime \prime}$ would allow the measurement of the depletion factor (and estimate of the $\mathrm{CO} / \mathrm{dust}$ ratio) around the mid-plane while an angular resolution of $0.1^{\prime \prime}$ would in addition allow us to determine the shape of this area.

Besides angular resolution and local line width, inclination is another limitation of the method. To the first order, the disk should be edge-on to within $h(r) / r$ for the TRD to be directly useable, i.e., in the range $80-90^{\circ}$ given the typical $h(r) / r$ of disks 


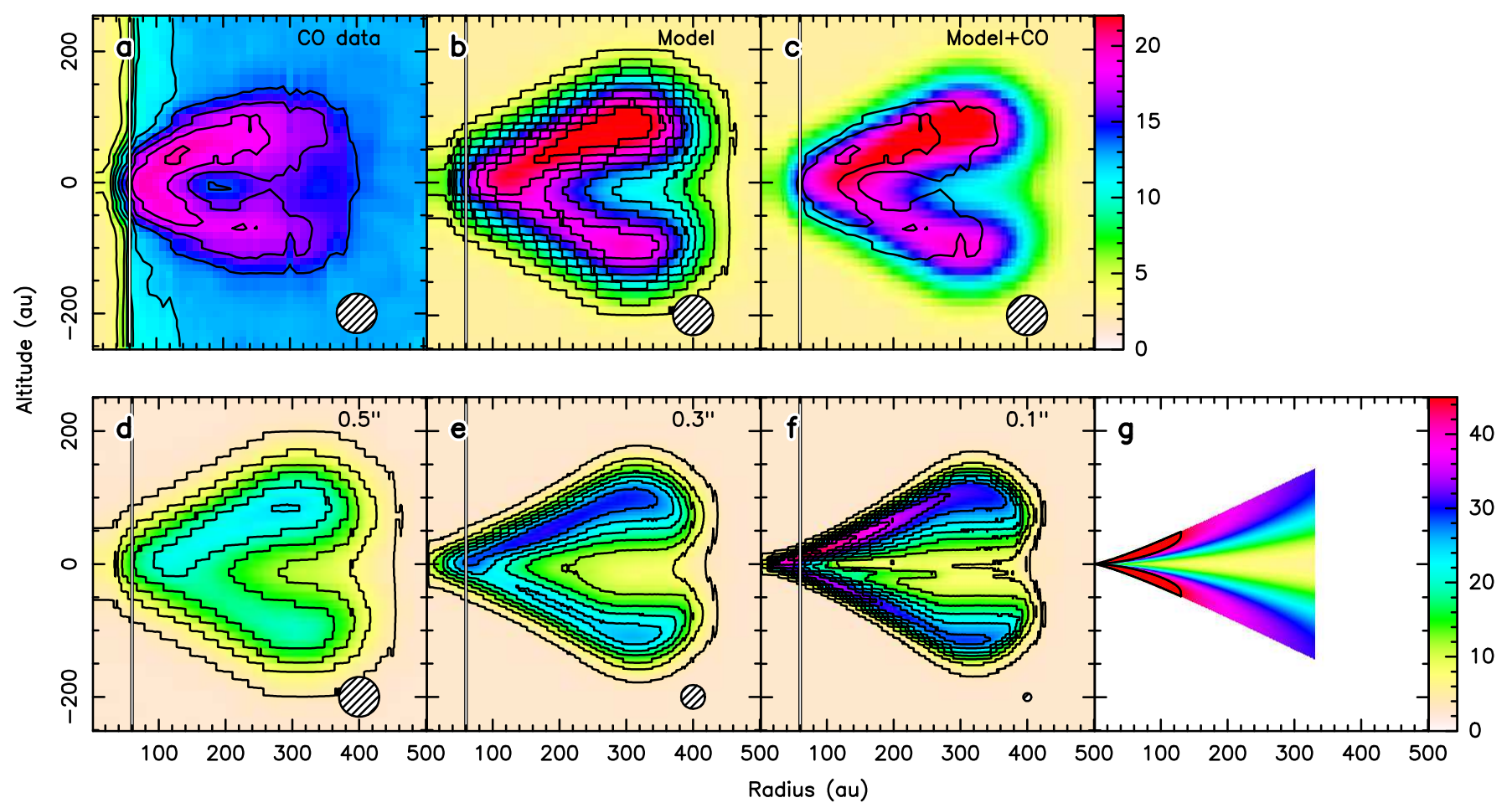

Fig. 8. TRD for CO $J=2-1$ (in Kelvin). $a$ ) Observed CO TRD; $b$ ) best model; $c$ ) CO TRD in false color with best model in black contours; d) model at $0.5^{\prime \prime}$ or 60 au resolution; $e$ ) model at $0.3^{\prime \prime}$ or 36 au resolution and $f$ ) at $0.1^{\prime \prime}(12 \mathrm{au})$ resolution, presented with the same color scale (contours in $4 \mathrm{~K}$ steps); $g$ ) initial temperature distribution (black contour at $45 \mathrm{~K}$ ).

at 100-300 au. However, even for somewhat lower inclination, the TRD can give insight into the location of the molecular layer. We illustrate this in Appendix B.

These simulations clearly demonstrate how well ALMA can characterize the structure of an edge-on protoplanetary disk, provided its distance is reasonable.

\section{Summary}

We report an analysis of the CO $J=2-1$ and CS $J=5-4$ ALMA maps of the Flying Saucer, a nearly edge-on protoplanetary disk orbiting a T Tauri star located in the $\rho$ Oph molecular cloud. At the angular resolution of $0.5^{\prime \prime}$ (60 au at $120 \mathrm{pc}$ ) and in spite of some confusion in $\mathrm{CO}$ due to the background molecular clouds, we find the following:

- the disk is in Keplerian orbit around a $0.57 M_{\odot}$ star and nearly edge-on (inclined by $87^{\circ}$ ). It does not exhibit a significant departure from symmetry in $\mathrm{CO}$ or in $\mathrm{CS}$;

- direct evidence of a vertical temperature gradient is demonstrated by the $\mathrm{CO}$ emission pattern; however, quantitative estimates are limited by the spatial resolution;

- distinguishing between $\mathrm{CO}$ depletion and very low temperatures is not possible because of the limited angular resolution. Models with $\mathrm{CO}$ depletion in the mid-plane only agree marginally better, and the mid-plane temperature cannot be significantly larger than $10 \mathrm{~K}$ at $100 \mathrm{au}$;

- the CO emission is observed between 1 and 3 scale heights, while the CS emission is located around one scale height. Subthermal excitation of CS may explain this apparent difference;

- CO is also observed beyond a radius of 230-260 au, in agreement with models predicting a secondary increase of temperature due to higher UV flux penetration in the outer disk.
However, the limited angular resolution does not rule out an alternate explanation with a molecular gap near $185 \mathrm{au}$.

Finally, our results demonstrate that observing an edge-on disk is a powerful method of directly sampling the vertical structure of protoplanetary disks provided the angular resolution is high enough. At least one data set with angular resolution around $0.1-0.2^{\prime \prime}$ is needed for a source located at $120-150 \mathrm{pc}$.

Acknowledgements. We thank the referee for constructive comments. This work was supported by "Programme National de Physique Stellaire" (PNPS from INSU/CNRS). This research made use of the SIMBAD database, operated at the CDS, Strasbourg, France. This paper makes use of the following ALMA data: ADS/JAO.ALMA\#2013.1.00387.S. ALMA is a partnership of ESO (representing its member states), NSF (USA), and NINS (Japan), together with NRC (Canada), NSC and ASIAA (Taiwan), and KASI (Republic of Korea) in cooperation with the Republic of Chile. The Joint ALMA Observatory is operated by ESO, AUI/NRAO, and NAOJ. This paper is based on observations carried out with the IRAM $30 \mathrm{~m}$ telescope. IRAM is supported by INSU/CNRS (France), MPG (Germany), and IGN (Spain). V.W.'s research is funded by the European Research Council (Starting Grant 3DICE, grant agreement 336474).

\section{References}

ALMA Partnership, Brogan, C. L., Pérez, L. M., et al. 2015, ApJ, 808, L3 Andrews, S. M., Wilner, D. J., Hughes, A. M., et al. 2012, ApJ, 744, 162 Andrews, S. M., Wilner, D. J., Zhu, Z., et al. 2016, ApJ, 820, L40 Beckwith, S. V. W., \& Sargent, A. I. 1993, ApJ, 402, 280

Bergin, E. A., Cleeves, L. I., Gorti, U., et al. 2013, Nature, 493, 644

Chapillon, E., Guilloteau, S., Dutrey, A., Piétu, V., \& Guélin, M. 2012, A\&A, 537, A60

Cleeves, L. I. 2016, ApJ, 816, L21

Cleeves, L. I., Öberg, K. I., Wilner, D. J., et al. 2016, ApJ, 832, 110

D’Alessio, P., Calvet, N., Hartmann, L., Lizano, S., \& Cantó, J. 1999, ApJ, 527, 893

Dartois, E., Dutrey, A., \& Guilloteau, S. 2003, A\&A, 399, 773

de Gregorio-Monsalvo, I., Ménard, F., Dent, W., et al. 2013, A\&A, 557, A133

Dutrey, A., Wakelam, V., Boehler, Y., et al. 2011, A\&A, 535, A104 
A\&A 607, A130 (2017)

Gorti, U., Hollenbach, D., Najita, J., \& Pascucci, I. 2011, ApJ, 735, 90

Grosso, N., Alves, J., Wood, K., et al. 2003, ApJ, 586, 296

Guilloteau, S., \& Dutrey, A. 1998, A\&A, 339, 467

Guilloteau, S., Dutrey, A., Piétu, V., \& Boehler, Y. 2011, A\&A, 529, A105

Guilloteau, S., Piétu, V., Chapillon, E., et al. 2016, A\&A, 586, L1

Lim, T.-H., Jo, Y.-S., Seon, K.-I., \& Min, K.-W. 2015, MNRAS, 449, 605

Loinard, L., Torres, R. M., Mioduszewski, A. J., \& Rodríguez, L. F. 2008, ApJ, 675, L29

Piétu, V., Dutrey, A., \& Guilloteau, S. 2007, A\&A, 467, 163

Pontoppidan, K. M., Stapelfeldt, K. R., Blake, G. A., van Dishoeck, E. F., \& Dullemond, C. P. 2007, ApJ, 658, L111

Qi, C., Ho, P. T. P., Wilner, D. J., et al. 2004, ApJ, 616, L11
Qi, C., Öberg, K. I., Wilner, D. J., et al. 2013, Science, 341, 630

Reboussin, L., Guilloteau, S., Simon, M., et al. 2015, A\&A, 578, A31

Rosenfeld, K. A., Qi, C., Andrews, S. M., et al. 2012, ApJ, 757, 129

Rosenfeld, K. A., Andrews, S. M., Hughes, A. M., Wilner, D. J., \& Qi, C. 2013, ApJ, 774, 16

Schwarz, K. R., Bergin, E. A., Cleeves, L. I., et al. 2016, ApJ, 823, 91

Teague, R., Guilloteau, S., Semenov, D., et al. 2016, A\&A, 592, A49

Teague, R., Semenov, D., Gorti, U., et al. 2017, ApJ, 835, 228

Thi, W.-F., Mathews, G., Ménard, F., et al. 2010, A\&A, 518, L125

van Boekel, R., Henning, T., Menu, J., et al. 2017, ApJ, 837, 132

Weidenschilling, S. J. 1977, MNRAS, 180, 57 


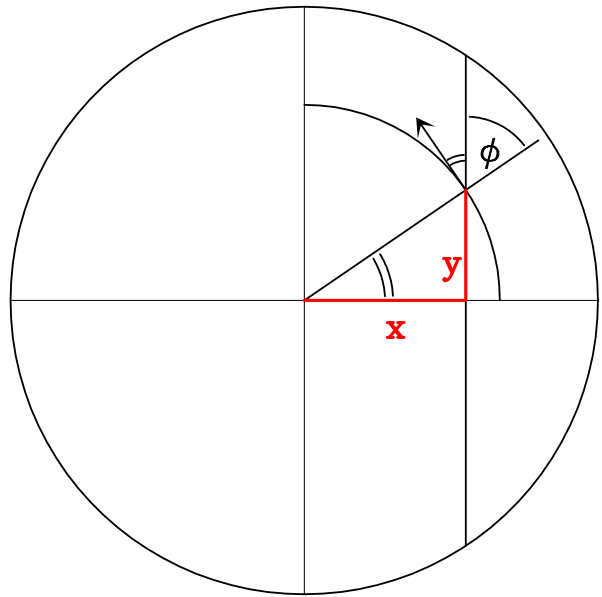

Fig. A.1. Definition of notations.

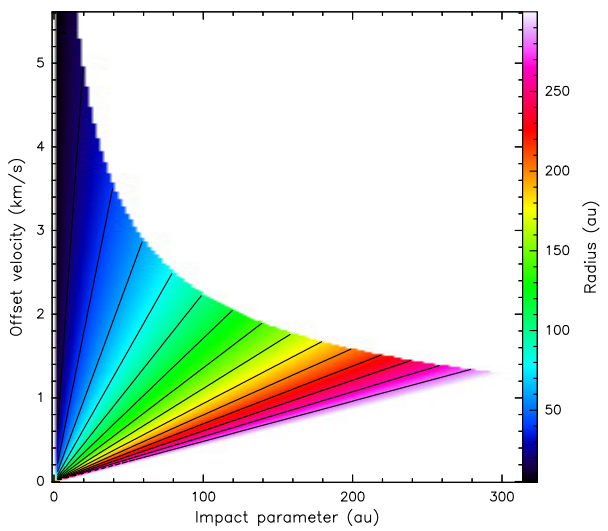

Fig. A.2. Radius as a function of position and velocity for an edge-on Keplerian disk

\section{Appendix A: position-velocity diagram for an edge-on Keplerian disk}

Let $x$ be the impact parameter in the disk, $y$ the coordinate along the line of sight, and $r$ the radial distance

$r(y)=\sqrt{x^{2}+y^{2}}$,

where $y<y_{m}$, with $y_{m}$ given by

$y_{m}=\sqrt{R_{\mathrm{d}}^{2}-x^{2}}$

The angle $\phi$ is defined such that $x=r \sin \phi$ (see Fig. A.1). The projected velocity along the line of sight is

$V_{y}=\sqrt{G M / r} \sin \phi=\sqrt{G M / r} \frac{x}{r}=\sqrt{G M} \frac{x}{r^{3 / 2}}$
Thus we simply recover $r$

$r=\left[G M\left(\frac{x}{V_{y}}\right)^{2}\right]^{1 / 3}=R_{\mathrm{d}}\left(\frac{X}{V}\right)^{2 / 3}$,

where $X=x / R_{\mathrm{d}}, V=V_{y} / V_{\mathrm{d}}$, and $V_{\mathrm{d}}=\sqrt{G M / R_{\mathrm{d}}}$ is the rotation velocity at the outer disk radius. Since $x \leq r \leq R_{\mathrm{d}}$, the above equation has a solution provided $X<V<1 / \sqrt{X}$ (i.e., $V_{y}>V_{\mathrm{d}} x / R_{\mathrm{d}}$, and $V_{y}<\sqrt{G M / x}$ ). So, for any given velocity $V_{y}$ and impact parameter $x$, we can solve for $r$, and then recover $y= \pm \sqrt{r^{2}-x^{2}}$ along the line of sight.

As a consequence, in the PV diagram (showing functions of $(x, V))$ of a Keplerian disk, any line starting from $(x=0, V=$ $\left.V_{\text {sys }}\right)$ represents locii of constant radius. This is illustrated in Fig. A.2. We use this property to directly recover the temperature as a function of radius (by taking the mean or the median for any given $r$ ) and altitude (by making cuts in the PV diagrams for different altitudes).

For optically thick lines, this procedure yields the (beam averaged) excitation temperature, and thus the kinetic temperature if the line is thermalized.

For optically thin lines, the opacity is a function of $(r, x)$ because of the Keplerian shear which breaks the rotational symmetry. The above procedure thus yields a more complex function of the temperature and density, those values, however, cannot exceed the excitation temperature at any radius $r$.

\section{Appendix B: Inclination effects}

Figure B.1 shows the expected TRDs of our fiducial disk model (Table 2) for different disk inclinations. In this model, CO emits mostly 1 or 2 scale heights above the disk mid-plane, thus when the inclination differs from edge-on by more than $h(r) / r$, the two opposite layers can project on the same side compared to the mid-plane projection. The farthest part of the disk projects to positive altitudes in Fig. B.1. It appears warmer than the projection of the nearest part because for the same impact parameter in altitude, the line of sight intercepts first warm gas due to the disk flaring; for the nearest part, the warm gas is hidden behind the forefront colder regions (see Dartois et al. 2003). The depletion in the mid-plane makes a clear distinction between the two emitting cones in the disk, resulting in a bright double layer at positive altitudes for $i=70-75^{\circ}$. At lower inclinations, the projected velocity gradient becomes insufficient to clearly separate these two layers on the TRD. For $i=80-85^{\circ}$, the two layers no longer project on the same side: a small lukewarm "finger" of emission appears at an altitude around $-20 \mathrm{au}$. 


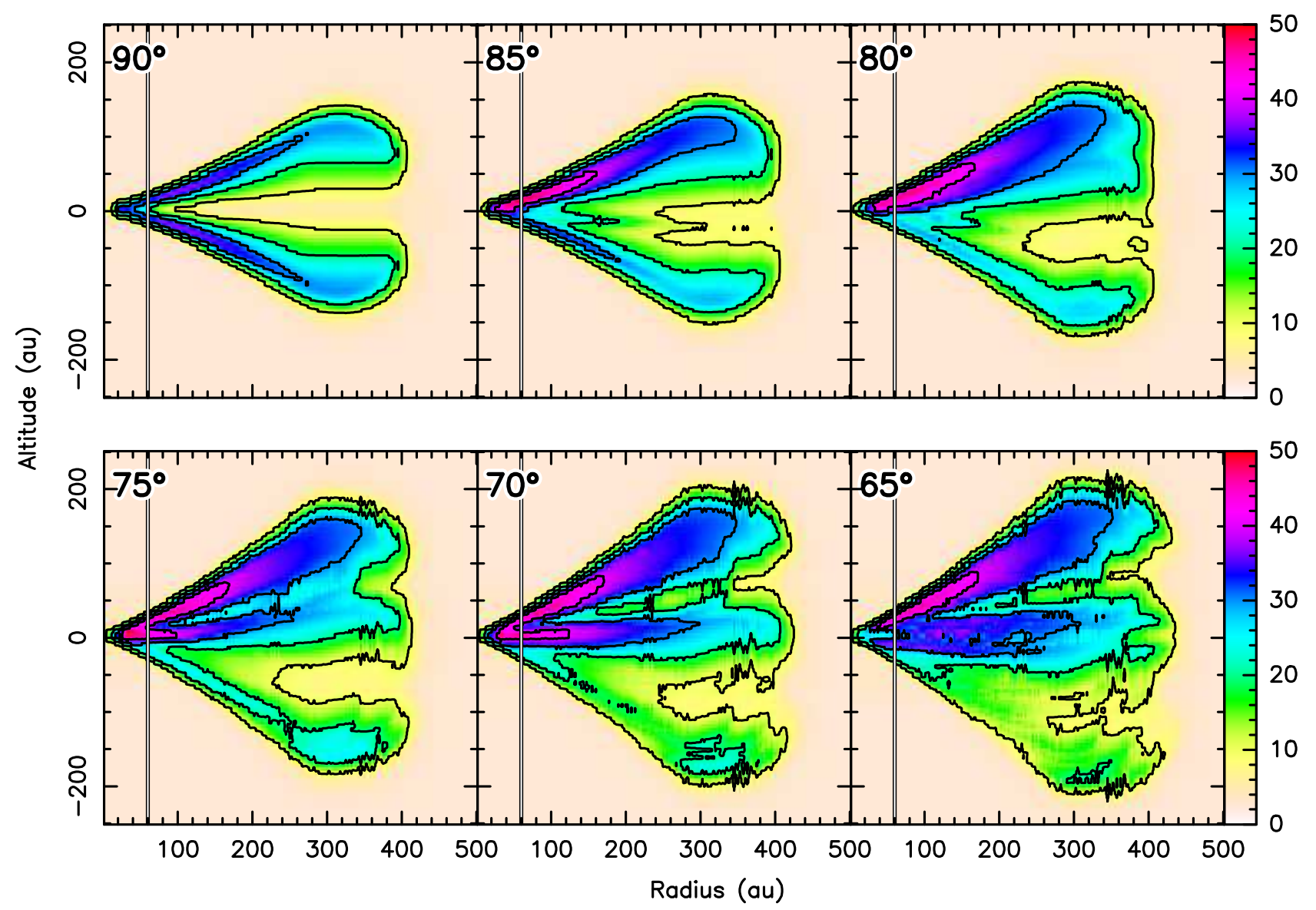

Fig. B.1. TRD for $\mathrm{CO} J=2-1$ (in kelvin) of our best disk model for different inclinations. Contours are in $10 \mathrm{~K}$ steps. 